\title{
Dichotomy Result on 3-Regular Bipartite Non-negative Functions
}

\author{
Austen Z. Fan* \\ zfan64@wisc.edu
}

\author{
Jin-Yi Cai ${ }^{\dagger}$ \\ jyc@cs.wisc.edu
}

\begin{abstract}
We prove a complexity dichotomy theorem for a class of Holant problems on 3-regular bipartite graphs. Given an arbitrary nonnegative weighted symmetric constraint function $f=$ $\left[x_{0}, x_{1}, x_{2}, x_{3}\right]$, we prove that the bipartite Holant problem Holant $\left(f \mid\left(=_{3}\right)\right)$ is either computable in polynomial time or \# $\mathrm{P}$-hard. The dichotomy criterion on $f$ is explicit.
\end{abstract}

\section{Introduction}

Holant problems are also called edge-coloring models. They can express a broad class of counting problems, such as counting matchings (\#MATCHINGS), perfect matchings (\#PM), edge-colorings, cycle coverings, and a host of counting orientation problems such as counting Eulerian orientations.

Given an input graph $G=(V, E)$, we identify each edge $e \in E$ as a variable over some finite domain $D$, and identify each vertex $v$ as a constraint function $f_{v}$. Then the partition function is the following sum of product $\sum_{\sigma: E \rightarrow D} \prod_{v \in V} f_{v}\left(\left.\sigma\right|_{E(v)}\right)$, where $E(v)$ denotes the edges incident to $v$ and $f_{v}\left(\left.\sigma\right|_{E(v)}\right)$ is the evaluation of $f_{v}$ on the restriction of $\sigma$ on $E(v)$. For example, \#MATChInGS and \#PM are counting problems specified by the constraint function AT-MosT-One, respectively ExACT-OnE, which outputs value 1 if the input bits have at most one 1, respectively exactly one 1 , and outputs 0 otherwise. Thus, every term $\prod_{v \in V} f_{v}\left(\left.\sigma\right|_{E(v)}\right)$ evaluates to 1 or 0 , and is 1 iff the assignment $\sigma$ is a mathching, respectively a perfect mathching. The framework of Holant problems is intimately related to Valiant's holographic algorithms [16]. Holant problems can encompass all counting constraint satisfaction problems (\#CSP), in particular all vertex-coloring models from statistical physics. For \#CSP, Bulatov [3] proved a sweeping complexity dichotomy. Dyer and Richerby [9] gave another proof of this dichotomy and also showed that the dichotomy is decidable. Cai, Chen, and $\mathrm{Lu}$ in [6] extended this to nonnegative weighted case. This was further generalized to complex weighted \#CSP [5]. However, no full dichotomy has been proved for Holant problems.

Every \#CSP problem can be easily expressed as a Holant problem. On the other hand, Freedman, Lovász and Schrijver [10] proved that the prototypical Holant problem \#PM cannot be expressed as a vertex-coloring model using any real constraint function, and this was extended to complex constraint functions [7]. Thus Holant problems are strictly more expressive.

\footnotetext{
* Department of Computer Sciences, University of Wisconsin-Madison.

${ }^{\dagger}$ Department of Computer Sciences, University of Wisconsin-Madison. Supported by NSF CCF-1714275.
} 
In this paper we consider the Boolean domain $D=\{0,1\}$. Significant knowledge has been gained about the complexity of Holant problems $[1,2,4,8,13,14]$. However, there has been very limited progress on bipartite Holant problems where the input graph $G=(U, V, E)$ is bipartite, and every constraint function on $U$ and $V$ comes from two separately specified sets of constraint functions. This is not an oversight; the reason is a serious technical obstacle. When the graph is bipartite and, say, $r$-regular, there is a curious number theoretic limitation as to what types of subgraph fragments, called gadgets, one can possibly construct. It turns out that every constructible gadget must have a rigid arity restriction; e.g., if the gadget represents a constraint function that can be used for a vertices in $U$ or in $V$, the arity (the number of input variables) of the function must be congruent to 0 modulo $r$.

We initiate in this paper the study of Holant problems on bipartite graphs. To be specific, we classify Holant problems on 3-regular bipartite graphs where vertices of one side are labeled with a nonnegative weighted symmetric constraint function $f=\left[x_{0}, x_{1}, x_{2}, x_{3}\right]$, which takes value $x_{0}, x_{1}, x_{2}, x_{3}$ respectively when the input has Hamming weight $0,1,2,3$; the vertices of the other side are labeled by the ternary equality function $\left(=_{3}\right)$. Such graphs can be viewed as incidence graphs of hypergraphs. Thus one can interpret problems of this type as on 3-uniform (every hyperedge has size 3) 3-regular (every vertex appears in 3 hyperedges) hypergraphs. In this view, e.g., counting perfect matchings on 3-uniform hypergraphs (the number of subsets of hyperedges that cover every vertex exactly once) corresponds to the ExACT-OnE function $[0,1,0,0]$. Alternatively one can think of them as set systems where every set has 3 elements and every element is in 3 subsets, and we count the number of exact-3-covers (\#X3C). Denote the problem of computing the partition function in this case as Holant $(f \mid(=3))$. We prove that for all $f$, this problem is either computable in polynomial time or \#P-hard, depending on an explicit dichotomy criterion on $x_{0}, x_{1}, x_{2}, x_{3}$. Suppose $(X, \mathcal{S})$ is a 3 -uniform set system where every $x \in X$ appears in 3 sets in $\mathcal{S}$. In the $0-1$ case, this dichotomy completely classifies the complexity of counting the number of ways to choose $\mathcal{S}^{\prime} \subseteq \mathcal{S}$ while satisfying some local constraint specified by $f$. In the more general nonnegative weighted case, this is to compute a weighted sum of products.

As mentioned earlier the main technical obstacle to this regular bipartite Holant dichotomy is the number theoretic arity restriction. We overcome this obstacle by considering straddled constraint functions, i.e., those functions which have some input variables that must be connected to one side of the bipartite graph while some other variables must be connected to the other side. Then we introduce a lemma that let's interpolate a degenerate constraint function by iterating the straddled function construction. Using a Vandermonde system we can succeed in this interpolation. Typically in proving a Holant dichotomy, getting a degenerate constraint function is useless and signifies failure. But here we turn the table, and transform this "failure" to a "success" by "peel" off a constraint function which is a tensor factor, whereby to break the number theoretic arity restriction.

This paper is a mere starting point for understanding bipartite Holant problems. Almost every generalization is an open problem at this point, including more than one constraint function on either side, other regularity parameter $r$, real or complex valued constraint functions which allow cancellations, etc. The bigger picture is to gain a systematic understanding of all such counting problems in a classification program, a theme seems second to none in its centrality to counting complexity theory, short of proving $\mathrm{PF} \neq \# \mathrm{P}$. 


\section{Preliminaries}

In this paper we consider the following subclass of Holant problems. An input 3-regular bipartite graph $G=(U, V, E)$ is given, where each vertex on $V$ is assigned the EquALITY of arities $3(=3)$ and each vertex on $U$ is assigned a ternary symmetric constraint function (also called a signature) $f$ with nonnegative values. The problem is to compute the quantity

$$
\operatorname{Holant}(G)=\sum_{\sigma: E \rightarrow\{0,1\}} \prod_{u \in U} f\left(\left.\sigma\right|_{E(u)}\right) \prod_{v \in V}\left(={ }_{3}\right)\left(\left.\sigma\right|_{E(v)}\right)
$$

Equivalently, this can be stated as a weighted counting constraint satisfaction problem on Boolean variables defined on 3-regular bipartite graphs: The input is a 3-regular bipartite $G=$ $(U, V, E)$, where every $v \in V$ is a Boolean variable and every $u \in U$ represents the nonnegative valued constraint function $f$. An edge $(u, v) \in E$ indicates that $v$ appears in the constraint at $u$. Being 3-regular means that every constraint has 3 variables and every variable appears in 3 constraints.

We adopt the notation $f=\left[x_{0}, x_{1}, x_{2}, x_{3}\right]$ to represent the ternary symmetric signature $f$ where $f(0,0,0)=x_{0}, f(0,0,1)=f(0,1,0)=f(1,0,0)=x_{1}, f(0,1,1)=f(1,0,1)=f(1,1,0)=x_{2}$ and $f(1,1,1)=x_{3}$. The Equality of arities 3 is $(=3)=[1,0,0,1]$. For clarity, we shall call vertices in $V$ are on the right hand side (RHS) and vertices in $U$ are on the left hand side (LHS). We denote this problem Holant $(f \mid(=3))$.

A gadget, such as those illustrated in Figure 1 , is a bipartite graph $G=\left(U, V, E_{\text {in }}, E_{\text {out }}\right)$ with internal edges $E_{\text {in }}$ and dangling edges $E_{\text {out }}$. There can be $m$ dangling edges internally incident to vertices from $U$ and $n$ dangling edges internally incident to vertices from $V$. These $m+n$ dangling edges correspond to Boolean variables $x_{1}, \ldots, x_{m}, y_{1}, \ldots, y_{n}$ and the gadget defines a signature

$$
f\left(x_{1}, \ldots, x_{m}, y_{1}, \ldots, y_{n}\right)=\sum_{\sigma: E_{\mathrm{in}} \rightarrow\{0,1\}} \prod_{u \in U} f\left(\left.\widehat{\sigma}\right|_{E(u)}\right) \prod_{v \in V}\left(=_{3}\right)\left(\left.\widehat{\sigma}\right|_{E(v)}\right)
$$

where $\widehat{\sigma}$ denotes the extension of $\sigma$ by the assignment on the dangling edges.

To preserve the bipartite structure, we must be careful in any gadget construction how each external wire is to be connected, i.e., as an input variable whether it is on the LHS (like those of $f$ which can be used to connect to $\left(=_{3}\right)$ on the RHS), or it is on the RHS (like those of $\left(=_{3}\right)$ which can be used to connect to $f$ on the LHS). See illustrations in Figure 1.

If a gadget construction produces a constraint function $g$ such that all of its variables are on the LHS. We claim that its arity must be a multiple of 3 . This follows from the following more general statement that if a constraint function has $n$ input variables on the LHS and $m$ input variables on the RHS, then $n \equiv m \bmod 3$. This can be easily proved by induction on the number of occurrences of $f$ and $\left(=_{3}\right)$ in a gadget construction $\Gamma$ : If either $f$ or $\left(=_{3}\right)$ do not occur, then the function is a tensor product of $\left(=_{3}\right)$ or $\left(=_{3}\right)$, thus clearly of arity $n \equiv 0 \bmod 3$ or $m \equiv 0 \bmod 3$. Suppose $f$ or $(=3)$ both occur and let $x$ be an external dangling edge. It is internally connected to a vertex $v$, which is labeled either $f$ (if $v \in U$ ) or $\left(=_{3}\right.$ ) (if $v \in V$ ). Let $v$ have exactly $k \in\{1,2,3\}$ incident edges that are dangling edges. Now we remove $v$ and get a gadget $\Gamma^{\prime}$ with fewer occurrences of $f$ and $\left(=_{3}\right)$. The induction hypothesis and a simple accounting of the arity for variables on the LHS and the RHS separately complete the induction.

One idea that is instrumental in this paper is to use gadgets that produce straddled signatures. For example the gadget $G_{1}$ in Figure 1(a) has one variable on the LHS and one variable on the 
RHS. Such gadgets can be iterated while respecting the bipartite structure. The signature matrix of such straddled gadgets will adopt the notation that row indices denote the input from LHS and column indices denote the input from RHS. For example, the signature matrix for $G_{1}$ in Figure 1(a) where we place $\left[x_{0}, x_{1}, x_{2}, x_{3}\right]$ on the square and $\left(=_{3}\right)$ on the circle will be $\left(\begin{array}{ll}x_{0} & x_{2} \\ x_{1} & x_{3}\end{array}\right)$. A binary signature is degenerate if it has determinant 0 . We will use such constructions to interpolate a degenerate straddled signature so that we can "split" it to get unary signatures. To justify that we can indeed "split" a degenerate straddled signature, whenever we connect a unary signature, we have to "use up" another unary signature, for the pair was created by that degenerate straddled signature. Intuitively, we can "use all other edges up" by connecting them to form known positive global factors of the Holant value. This intuition is encoded into the following lemma. Effectively, we can split a degenerate binary straddled signature into unary signatures to be freely used.

Lemma 2.1. Let $f$ and $g$ be two nonnegative valued signatures. If a degenerate nonnegative binary straddled signature $\left(\begin{array}{cc}1 & x \\ y & x y\end{array}\right)$ can be interpolated in the problem Holant $(f \mid g)$, then

$$
\text { Holant }(f \mid\{g,[1, x]\}) \leq_{T} \operatorname{Holant}(f \mid g) .
$$

A similar statement holds for adding the unary $[1, y]$ on the LHS.

Remark: The same proof applies for $\left(\begin{array}{cc}y & x y \\ 1 & x\end{array}\right)$ to get unary signatures $[1, x]$ on the RHS, or unary $[y, 1]$ on the LHS.

Proof. We prove (2.1). Let $f$ and $g$ have arity $m$ and $n$ respectively. We may assume that $g$ is not a multiple of $[0,1]^{\otimes n}$ (including identically 0 ), for otherwise Holant $(f \mid\{g,[1, x]\})$ can be computed in PF, since all signatures on the RHS are degenerate and can be applied directed as unary signatures on copies of $f$.

Let $k=\operatorname{gcd}(m, n)$, and $s=n / k \geq 1$. Consider any bipartite signature grid $\Omega=(G, \pi)$ for Holant $(f \mid\{g,[1, x]\})$. Let $N_{f}, N_{g}, N_{u}$ be the numbers of occurrences of $f, g,[1, x]$ respectively. Then we have

$$
m N_{f}=n N_{g}+N_{u},
$$

thus $N_{u} \equiv 0 \bmod k$. Let $t=N_{u} / k \geq 0$. We may assume $t \geq 1$, for otherwise $[1, x]$ does not occur and the reduction is trivial.

We will compute (Holant $(G))^{s}$, the $s$-th power of the value Holant $(G)$, using an oracle for Holant $(f \mid g)$. Since the value Holant $(G)$ is nongenative, we can obtain it from $(\operatorname{Holant}(G))^{s}$.

In $G$, we replace each occurrence of $[1, x]$ with the binary straddled signature $\left(\begin{array}{cc}1 & x \\ y & x y\end{array}\right)$, with one end connected to LHS, and leaving one edge yet to be connected to RHS. This creates a total of $N_{u}$ such edges. This is equivalent to connecting $N_{u}$ copies of the unary signature $[1, x]$ to LHS, and having $N_{u}$ copies of the unary signature $[1, y]$ yet to be connected to RHS. Now in $s$ disjoint copies of $\Omega$ there will be $s N_{u}=s t k$ copies of $[1, y]$ to be connected, to which we create $t$ copies of $g$. In other words we take $g^{\otimes t}$ with total arity $s t k$ and connect all $s t k$ unary signatures $[1, y]$ to it. Since $y \geq 0$ and $g$ is not a multiple of $[0,1]^{\otimes n}$, we get an easily computable positive factor. 


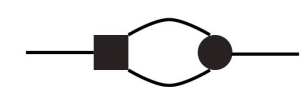

(a) Binary straddled gadget $G_{1}$

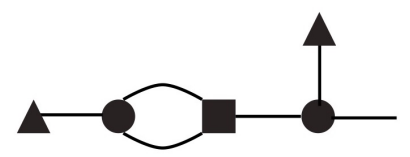

(c) Unary gadget $G_{3}$

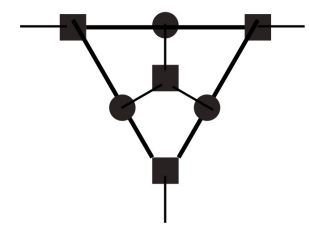

(b) Ternary gadget $G_{2}$

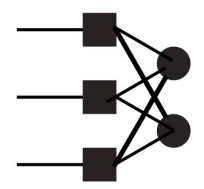

(d) Ternary gadget $G_{4}$

Figure 1: Some gadgets

The main result of this paper is the following:

Theorem 2.2. Holant $\left(\left[x_{0}, x_{1}, x_{2}, x_{3}\right] \mid\left(=_{3}\right)\right)$ where $x_{i} \geq 0$ for $i=0,1,2,3$ is \#P-hard except in the following cases, for which the problem is in $\mathrm{FP}$.

1. $\left[x_{0}, x_{1}, x_{2}, x_{3}\right]$ is degenerate;

2. $x_{1}=x_{2}=0$;

3. $\left[\left(x_{1}=x_{3}=0\right) \wedge\left(x_{0}=x_{2}\right)\right]$ or $\left[\left(x_{0}=x_{2}=0\right) \wedge\left(x_{1}=x_{3}\right)\right]$.

In case 1 the signature $\left[x_{0}, x_{1}, x_{2}, x_{3}\right]$ decomposes into three unary signatures. In case 2 $\left[x_{0}, 0,0, x_{3}\right]$ is a generalized equality. In case 3 the signature is in the affine class; see more details about these tractable classes in [4]. Therefore the Holant problem is in FP in cases 1-3. The main claim lies in that all other cases are \#P-hard. When proving our main result, we shall apply the following dichotomy theorem on 2-3 Holant problem [12]:

Theorem 2.3. Suppose $a, b \in \mathbb{C}$, and let $X=a b, Z=\left(\frac{a^{3}+b^{3}}{2}\right)^{2}$. Then Holant $([a, 1, b] \mid(=3))$ is \# $\mathrm{P}$-hard except in the following cases, for which the problem is in $\mathrm{P}$.

1. $X=1$;

2. $X=Z=0$;

3. $X=-1$ and $Z=0$;

4. $X=-1$ and $Z=-1$.

In fact, since this paper mainly concerns with nonnegative valued functions, when establishing \#P-hardness we usually only need to consider the exceptional case $X=1$.

\section{Interpolation From A Binary Straddled Signature}

When $x_{0}$ and $x_{3}$ are not both 0 , we can normalize the signature. If $x_{0} \neq 0$ we divide the signature by $x_{0}$, and get the form $[1, a, b, c]$, with $a, b, c \geq 0$. If $x_{0}=0$, but $x_{3} \neq 0$, we can flip all 0 and 1 inputs, which amounts to a reversal of the signature and get the above form. This does not change the complexity since the Holant value is only modified by a known nonzero factor. 
Consider arguably the simplest possible gadget $G_{1}$ in Figure 1 . We have the signature matrix $G_{1}=\left(\begin{array}{ll}1 & b \\ a & c\end{array}\right)$. Let $\Delta=\sqrt{(1-c)^{2}+4 a b}$, and assume for now $\Delta \neq 0$, and we take the positive square root. Note that $\Delta=0$ iff $(c=1) \wedge(a b=0)$. The matrix $G_{1}$ has two distinct eigenvalues $\lambda=\frac{-\Delta+(1+c)}{2}$ and $\mu=\frac{\Delta+(1+c)}{2}$. If $a \neq 0$, let $x=\frac{\Delta-(1-c)}{2 a}$ and $y=\frac{\Delta+(1-c)}{2 a}$. The matrix for $G_{1}$ has the Jordan Normal Form

$$
\left(\begin{array}{ll}
1 & b \\
a & c
\end{array}\right)=\left(\begin{array}{cc}
-x & y \\
1 & 1
\end{array}\right)\left(\begin{array}{cc}
\lambda & 0 \\
0 & \mu
\end{array}\right)\left(\begin{array}{cc}
-x & y \\
1 & 1
\end{array}\right)^{-1}
$$

We now interpolate a binary degenerate straddled signature which will be used several times later.

Lemma 3.1. Given the binary straddled signature $G_{1}=\left(\begin{array}{ll}1 & b \\ a & c\end{array}\right)$ with $a \neq 0$ and $\Delta>0$, we can interpolate unary signatures $[1, x]$ on $R H S$ or $[y, 1]$ on $L H S$.

Proof. For $\Delta>0$, we have $x+y=\Delta / a>0$. Consider

$$
D=\frac{1}{x+y}\left(\begin{array}{cc}
y & x y \\
1 & x
\end{array}\right)=\left(\begin{array}{cc}
-x & y \\
1 & 1
\end{array}\right)\left(\begin{array}{ll}
0 & 0 \\
0 & 1
\end{array}\right)\left(\begin{array}{cc}
-x & y \\
1 & 1
\end{array}\right)^{-1} .
$$

Given any signature grid $\Omega$ where the binary degenerate straddled signature $D$ appears $n$ times, we form gadgets $G_{1}^{s}$ where $0 \leq s \leq n$ by iterating the $G_{1}$ gadget $s$ times and replacing each occurrence of $D$ with $G_{1}^{s}$. Denote the resulting signature grid as $\Omega_{s}$. We stratify the assignments in the Holant sum for $\Omega$ according to assignments to $\left(\begin{array}{cc}\lambda & 0 \\ 0 & \mu\end{array}\right)$ as:

- $(0,0) i$ times;

- $(1,1) j$ times;

with $i+j=n$; all other assignments will contribute 0 in the Holant sum. Let $c_{i, j}$ be the sum over all such assignments of the products of evaluations (including the contributions from $\left(\begin{array}{cc}-x & y \\ 1 & 1\end{array}\right)$ and its inverse). Then we have

$$
\operatorname{Holant}_{\Omega_{s}}=\sum_{i+j=n}\left(\lambda^{i} \mu^{j}\right)^{s} \cdot c_{i, j}
$$

and Holant $\Omega=c_{0, n}$. Since $\Delta>0$, the coefficients form a full rank Vandermonde matrix. Thus we can interpolate $D$ by solving the linear system of equations in polynomial time. Ignoring a nonzero factor, we may split $D$ into unary signatures $[y, 1]$ on LHS or $[1, x]$ on RHS.

We have $\Delta \geq|1-c|$, and $x, y \geq 0$. Thus we can separate $D$ and obtain unary signatures as specified in Lemma 2.1 .

The following lemma lets us interpolate unary signatures on the RHS from a binary gadget with a straddled signature and a suitable unary signature $s$ on the RHS. Mathematically, the proof is essentially the same as in [15], but technically Lemma 3.2 applies to binary straddled signatures.

Lemma 3.2. Let $M \in \mathbb{R}^{2 \times 2}$ be the signature matrix for a binary straddled gadget which is diagonalizable with distinct eigenvalues, and let $s=[a, b]$ be a unary signature on $R H S$ that is not a row eigenvector of $M$. Then $\left\{s \cdot M^{j}\right\}_{j \geq 0}$ can be used to interpolate any unary signature on RHS. 


\section{A Basic Lemma}

In this section, we prove Lemma 4.2 which will be invoked in the remaining cases.

When $\neg\left(x_{0}=x_{3}=0\right)$, we normalize the signature $\left[x_{0}, x_{1}, x_{2}, x_{3}\right]$ to be $[1, a, b, c]$, where $a, b, c \geq$ 0 . We first deal with a special case when $a=b$ and $c=1$, which will be used in the proof of Lemma 4.2. By a slight abuse of notation, we say $[1, a, b, c]$ is \#P-hard or in FP if the problem Holant $\left([1, a, b, c] \mid\left(=_{3}\right)\right)$ is \#P-hard or in $\mathrm{FP}$, respectively.

Lemma 4.1. $[1, a, a, 1]$ is \#P-hard unless $a=0$ or $a=1$ in which case the problem is in $\mathrm{FP}$.

Proof. When $a=0$ or 1 , the signature $[1, a, a, 1]$ is clearly in FP. Suppose $a \neq 0$, 1 , we have $\Delta=2 a>0$. By Lemma 3.1, we can interpolate the unary signature $[1, x]=[1,1]$ on RHS. Connect $[1,1]$ on RHS to $[1, a, a, 1]$ on LHS, we get the binary signature $[1+a, 2 a, 1+a]$ on LHS. Invoke Theorem 2.3, as $a \neq 1$, the problem $[1+a, 2 a, 1+a]$ is \#P-hard, and thus $[1, a, a, 1]$ is \#P-hard.

We are now ready to prove Lemma 4.2, the basic lemma.

Lemma 4.2. When $a b \neq 0$, the problem $[1, a, b, c]$ is \#P-hard unless it is degenerate in which case the problem is in $\mathrm{FP}$.

Proof. As $a b \neq 0$, we have $a \neq 0$, and $\Delta>0$. By Lemma 3.1, we can interpolate the unary signature $[1, x]$ on RHS or $[y, 1]$ on LHS where $x=\frac{\Delta-(1-c)}{2 a}$ and $y=\frac{\Delta+(1-c)}{2 a}$. Consider the unary gadget $G_{3}$ in Figure 1 where we place the circles to be $\left(=_{3}\right)$, the triangles to be $[y, 1]$, and the square to be $[1, a, b, c]$. It has the unary signature $\left[y^{2}+y b, y a+c\right]$ on the RHS. By Lemma 3.2, we can interpolate any unary gadget, in particular $\Delta_{0}:=[1,0]$ and $\Delta_{1}:=[0,1]$, on the RHS unless the signature of $G_{3}$ is a row eigenvector of $G_{1}$. By Equation (3.2), the row eigenvectors of $G_{1}$ are the rows of $\left(\begin{array}{cc}-x & y \\ 1 & 1\end{array}\right)^{-1}$, namely proportional to $[1,-y]$ and $[1, x]$. Since $y>0$ by $a b \neq 0$, the only exception is $\frac{y a+c}{y^{2}+y b}=x$.

Observe that $x y=\frac{b}{a}$. Solve for $y$ we get $\left(b-a^{2}\right) y=\left(a c-b^{2}\right)$. If $b=a^{2}$, then $a c=b^{2}$ and thus $[1, a, b, c]$ is degenerate. Otherwise, $y=\frac{a c-b^{2}}{b-a^{2}}$. Now plug into $y=\frac{\Delta+(1-c)}{2 a}$, we have $\Delta+(1-c)=\frac{2 a\left(a c-b^{2}\right)}{b-a^{2}}$ which implies $\left(a^{3}-b^{3}-a b(1-c)\right)(a b-c)=0$ (the high order multivariable polynomial magically factors out).

We now divide our discussion into three cases: (1) $\left[\left(a^{3}-b^{3}-a b(1-c) \neq 0\right) \wedge(a b-c \neq 0)\right]$, (2) $a^{3}-b^{3}-a b(1-c)=0$, and (3) $a b-c=0$.

Case 1: $\left(a^{3}-b^{3}-a b(1-c) \neq 0\right) \wedge(a b-c \neq 0)$

We can interpolate $\Delta_{0}$ and $\Delta_{1}$ on RHS. By connecting $\Delta_{0}$ and $\Delta_{1}$ on RHS to $[1, a, b, c]$ on LHS, we get the binary signatures $[1, a, b]$ and $[a, b, c]$ on LHS. That is we have

$$
\text { Holant }\left([1, a, b] \mid\left(==_{3}\right)\right) \leq_{T} \text { Holant }\left([1, a, b, c] \mid\left(=_{3}\right)\right)
$$

and

$$
\text { Holant }\left([a, b, c] \mid\left(=_{3}\right)\right) \leq_{T} \text { Holant }\left([1, a, b, c] \mid\left(={ }_{3}\right)\right) \text {. }
$$

By Theorem 2.3, the problem Holant $([1, a, b, c] \mid(=3))$ is \#P-hard unless $\frac{1}{a} \cdot \frac{b}{a}=1$ and $\frac{a}{b} \cdot \frac{c}{b}=1$, in which case the signature $[1, a, b, c]$ is degenerate and thus the problem is in FP.

Case 2: $a^{3}-b^{3}-a b(1-c)=0$ 
We have $1-c=\frac{a^{3}-b^{3}}{a b}$ and thus $\Delta=\sqrt{(1-c)^{2}+4 a b}=\frac{a^{3}+b^{3}}{a b}$. Thus the unary signature interpolated by Lemma 3.1 on RHS is $[1, x]=\left[1, \frac{\Delta-(1-c)}{2 a}\right]=\left[1, \frac{b^{2}}{a^{2}}\right]$. Connect $[1, x]$ on RHS to $[1, a, b, c]$ on LHS, we get the binary signature $\left[1+\frac{b^{2}}{a}, a+\frac{b^{3}}{a^{2}}, b+\frac{b^{2} c}{a^{2}}\right]$ on LHS. By Theorem 2.3, the problem $\left[1+\frac{b^{2}}{a}, a+\frac{b^{3}}{a^{2}}, b+\frac{b^{2} c}{a^{2}}\right]$ is \#P-hard and thus the problem $[1, a, b, c]$ is \#P-hard unless $\left(1+\frac{b^{2}}{a}\right)\left(b+\frac{b^{2} c}{a^{2}}\right)=\left(a+\frac{b^{3}}{a^{2}}\right)^{2}$, which, after substituting $c$, simplifies to $\left(a^{2}-b\right)\left(a^{3}+a b+2 b^{3}\right)=0$. Since $a, b>0$, we have $a^{3}+a b+2 b^{3} \neq 0$ and thus $a^{2}-b=0$. This combined with $a^{3}-b^{3}=a b(1-c)$ gives $c=a^{3}$, and thus $[1, a, b, c]$ is degenerate.

Case 3: $a b-c=0$

Observe that under this case we have $\Delta=1+c$. The unary signature interpolated on RHS is $[1, x]=\left[1, \frac{\Delta-(1-c)}{2 a}\right]=\left[1, \frac{c}{a}\right]=[1, b]$. We connect $[1, x]$ on RHS to $[1, a, b, c]$ on LHS to get binary signature $\left[1+a b, a+b^{2}, b+b c\right]$ on LHS. That is we have

$$
\text { Holant }\left(\left[1+a b, a+b^{2}, b+b c\right] \mid\left(==_{3}\right)\right) \leq_{T} \operatorname{Holant}\left([1, a, b, c] \mid\left(=_{3}\right)\right)
$$

By Theorem 2.3, this problem is \#P-hard unless $(1+a b)(b+b c)=\left(a+b^{2}\right)^{2}$ which implies $\left(a^{2}-b\right)\left(b^{3}-1\right)=0$. If $a^{2}-b=0$, since we are under the case $a b-c=0$, the signature $[1, a, b, c]$ is degenerate. If $b^{3}-1=0$, since $b$ is a positive real number, we have $b=1$. Thus the signature $[1, a, b, c]$ is simply $[1, a, 1, a]$.

Now consider the ternary gadget $G_{4}=\left[2+2 a^{3}, 2 a+2 a^{2}, 2 a+2 a^{2}, 2+2 a^{3}\right]$ on LHS where we place the circles to be $\left(=_{3}\right)$ and the squares to be $[1, a, 1, a]$. This signature by $G_{4}$ has the form in Lemma 4.1, and we see that it is \#P-hard unless $2+2 a^{3}=2 a+2 a^{2}$. So the problem $[1, a, 1, a]$ is \#P-hard and thus the problem $[1, a, b, c]$ is \#P-hard unless $a^{3}-a^{2}-a+1=(a-1)^{2}(a+1)=0$, which implies $a=1$. When $a=1$, the signature $[1, a, 1, a]$ is degenerate.

The proof is now complete.

We are now ready to prove a series of lemmas which lead to our main result, Theorem 2.2. The basic idea is to reduce cases to Lemma 4.2, and handle exceptional cases separately.

\section{$5 \quad$ Case $\neg\left(x_{0}=x_{3}=0\right)$}

With the help of Lemma 4.2 we can quickly finish this case. We may normalize $\left[x_{0}, x_{1}, x_{2}, x_{3}\right]$ to $[1, a, b, c]$ by dividing a nonzero factor, and flipping all input 0's and 1's if necessary (i.e., taking the reversal of the signature). The exceptional cases in Lemma 4.2 for $[1, a, b, c]$ are $a=0$ or $b=0$. We first consider the case $a \neq 0$ but $b=0$.

Lemma 5.1. If $a \neq 0$ and $b=0$, then $[1, a, b, c]$ is \#P-hard.

Proof. By gadget $G_{2}$ where we place the squares to be $[1, a, 0, c]$ and the circles to be $\left(={ }_{3}\right)$, we have a ternary signature $\left[1+3 a^{3}, a+a^{4}, a^{2}, a^{3}+c^{4}\right]$ on LHS. Thus Lemma 4.2 applies (after normalizing) and $\left[1+3 a^{3}, a+a^{4}, a^{2}, a^{3}+c^{4}\right]$ is \#P-hard unless it is degenerate, i.e., $\left(a+a^{4}\right)^{2}=\left(1+3 a^{3}\right)\left(a^{2}\right)$ and $\left(a^{2}\right)^{2}=\left(a+a^{4}\right)\left(a^{3}+c^{4}\right)$. The first equality forces $a=1$ for positive $a$. Then we get $\frac{1}{2}=1+c^{4} \geq 1$, which is a contradiction.

Now consider the case $a=0$. We have the following subcases (1) $c=1$, (2) $c=0$, and (3) $(c \neq 1) \wedge(c \neq 0)$. These are handled by the following three lemmas. 
Lemma 5.2. If $a=0$ and $c=1$, then $[1,0, b, 1]$ is \#P-hard unless $b=0$, in which case the problem is in FP.

Proof. By flipping 0 and 1 in the input, we equivalently consider the signature $[1, b, 0,1]$. This case is dealt with in Lemma 5.1 except when $b=0$ in which case it is in FP.

Lemma 5.3. If $a=0$ and $c=0$, then $[1,0, b, 0]$ is \#P-hard unless $b=0$ or $b=1$, in which case the problem is in $\mathrm{FP}$.

Proof. By gadget $G_{1}$ where we place $[1,0, b, 0]$ at the squares and $(=3)$ at the circles, we have the binary degenerate straddled signature $\left(\begin{array}{ll}1 & b \\ 0 & 0\end{array}\right)$. We can thus split and get unary signature $[1, b]$ on RHS. Connect $[1, b]$ on RHS and $[1,0, b, 0]$ on LHS, we get the binary signature $\left[1, b^{2}, b\right]$ on LHS. By Theorem 2.3, $\left[1, b^{2}, b\right]$ is \#P-hard unless $b=0$ or $b=1$. When $b=0$, the original signature $[1,0, b, 0]$ becomes $[1,0,0,0]$ which is degenerate and thus the problem is tractable. When $b=1$, the signature $[1,0, b, 0]$ becomes $[1,0,1,0]$ which is an affine signature and thus the problem is also tractable $([4]$ p.70).

Lemma 5.4. If $a=0, c \neq 0$, and $c \neq 1$, then $[1,0, b, c]$ is \#P-hard unless $b=0$, in which case the problem is in $\mathrm{FP}$.

Proof. By flipping 0 and 1 in the input, we equivalently consider the signature $[c, b, 0,1]$. Thus Lemma 5.1 applies after normalizing, except when $b=0$ in which case $[c, 0,0,1]$ is in FP.

The discussion for the case $\neg\left(x_{0}=x_{3}=0\right)$ is now complete.

\section{Case $x_{0}=x_{3}=0$}

If both $x_{1}=x_{2}=0$, the signature is identically zero and the problem is trivially in FP.

Suppose exactly one of $x_{1}$ and $x_{2}$ is 0 . In this case, by normalizing and possibly flipping 0 and 1 in the input, it suffices to consider the ternary signature $[0,1,0,0]$.

Lemma 6.1. The problem Holant $\left([0,1,0,0] \mid\left(=_{3}\right)\right)$ is \#P-hard.

Proof. We begin with a reduction from Restricted Exact Cover by 3-Set (RX3C) [11]. This is a restricted version of the Set Exact Cover problem where every set has exactly 3 elements and every element is in exactly 3 sets. Given any instance for RX3C, construct the 3-regular bipartite graph whose vertices on LHS are elements of the set $X$ and vertices on RHS are 3-element subsets of $X$. Connect an edge between one vertex $v$ on LHS to one vertex $C$ on RHS if and only if $v \in C$. Then every nonzero term in the Holant sum for Holant $\left([0,1,0,0] \mid\left(=_{3}\right)\right)$ exactly corresponds to one solution of RX3C. We observe that the reduction given in [11] (from the Exact Cover by 3 Set problem (X3C)) is parsimonious. And it is well known that SAT reduces to X3C parsimoniously via 3-Dimensional Matching (3DM). Thus we have the reduction chain:

$$
\# \mathrm{SAT} \leq_{T} \# 3 \mathrm{DM} \leq_{T} \# \mathrm{X} 3 \mathrm{C} \leq_{T} \# \mathrm{RX} 3 \mathrm{C} \leq_{T} \operatorname{Holant}\left([0,1,0,0] \mid\left(=_{3}\right)\right)
$$

We conclude that the problem $[0,1,0,0]$ is \#P-hard.

It remains to consider the case when $x_{1} \cdot x_{2} \neq 0$. We normalize the signature to be $[0,1, b, 0]$. 
Lemma 6.2. If $b \neq 0$, then the problem Holant $\left([0,1, b, 0] \mid\left(=_{3}\right)\right)$ is \#P-hard.

Proof. By gadget $G_{2}$ where we place the squares to be $[0,1, b, 0]$ and the circles to be $(=3)$, we have the ternary signature $\left[3 b^{2}, 1+2 b^{3}, 2 b+b^{4}, 3 b^{2}\right]$ on LHS. Lemma 4.2 applies (after normalizing) and the problem $\left[3 b^{2}, 1+2 b^{3}, 2 b+b^{4}, 3 b^{2}\right]$ is \#P-hard unless it is degenerate. The condition for $\left[3 b^{2}, 1+2 b^{3}, 2 b+b^{4}, 3 b^{2}\right]$ being degenerate is

$$
\left\{\begin{array}{l}
\frac{3 b^{2}}{1+2 b^{3}}=\frac{1+2 b^{3}}{2 b+b^{4}} \\
\frac{1+2 b^{3}}{2 b+b^{4}}=\frac{2 b+b^{4}}{3 b^{2}}
\end{array}\right.
$$

The second equation simplifies to $\left(b^{3}-1\right)^{2}=0$. Thus when $b \neq 1$, we conclude that $[0,1, b, 0]$ is \#P-hard.

Proceed with the case $[0,1,1,0]$. By gadget $G_{4}$, where we place the squares to be $[0,1,1,0]$ and the circles to be $[1,0,0,1]$, we get the (reversal invariant) ternary signature $[3,2,2,3]$ on LHS. By Lemma 4.2 this problem is \#P-hard.

We note that the problem Holant $\left([0,1,1,0] \mid\left(=_{3}\right)\right)$ when restricted to planar graphs is in FP.

Problem : Pl-\#HyperGragh-Moderate-3-Cover

Input : A planar 3-uniform 3-regular hypergraph $G$.

Output : The number of subsets of hyperedges that cover every vertex with no vertex covered three times.

This is exactly the problem Pl-Holant $([0,1,1,0] \mid(=3))$, the restriction of Holant $\left([0,1,1,0] \mid\left(==_{3}\right)\right)$ to planar graphs. Its P-time tractability is seen by the following holographic reduction using the Hadamard matrix $H=\left(\begin{array}{cc}1 & 1 \\ 1 & -1\end{array}\right)$ to counting weighted perfect matchings. Under this transformation both signatures $[0,1,1,0]$ and $\left(=_{3}\right)$ are transformed to matchgate signatures.

$$
H^{\otimes 3}(=3)=H^{\otimes 3}\left[\left(\begin{array}{l}
1 \\
0
\end{array}\right)^{\otimes 3}+\left(\begin{array}{l}
0 \\
1
\end{array}\right)^{\otimes 3}\right]=\left(\begin{array}{c}
1 \\
1
\end{array}\right)^{\otimes 3}+\left(\begin{array}{c}
1 \\
-1
\end{array}\right)^{\otimes 3}=[2,0,2,0],
$$

and

$$
[0,1,1,0]\left(H^{-1}\right)^{\otimes 3}=\frac{1}{8}\left[(1, \quad 1)^{\otimes 3}-(1, \quad 0)^{\otimes 3}-(0, \quad 1)^{\otimes 3}\right] H^{\otimes 3}=\frac{1}{4}[3,0,-1,0] .
$$

Since both these are matchgate signatures, the problem is reduced to counting weighted perfect matchings on planar graphs. Thus the planar problem Pl- Holant $\left([0,1,1,0] \mid\left(={ }_{3}\right)\right)$ can be computed in polynomial time using Kasteleyn's algorithm (see [16] and [4]). For readers unfamiliar with holographic algorithms, we will describe this algorithm for Pl-\#HYPERGRAGH-Moderate3-Cover in more detail in an Appendix.

\section{Acknowledgement}

We sincerely thank Shuai Shao for many helpful discussions. 


\section{Appendix}

The holographic algorithm for Pl-\#HYPERGRAGH-ModerATE-3-Cover is achieved by a many-tomany reduction from this problem to the problem of counting (weighted) planar perfect matchings (Pl-\#PM). Given planar weighted graph $G=(V, E, w)$ where $w: E \rightarrow \mathbb{R}$ is a weight function for the edge set, any perfect matching $M \subseteq E$ has weight $w(M)=\prod_{e \in M} w(e)$, and the partition function of the problem Pl-\#PM is

$$
\# \operatorname{PM}(G)=\sum_{\text {perfect matchings } M} w(M) .
$$

Notice that when all weights $w(e)=1$, i.e., in the unweighted case, $\# \operatorname{PM}(G)$ simply counts the number of perfect matchings in $G$. Kasteleyn's algorithm can compute this partition function $\# \mathrm{PM}(G)$ in polynomial time on planar graphs. It is important to note that Kasteleyn's algorithm can handle the case when $w(e)$ are both positive and negative real (even complex) numbers, which is important in this case for us.

The values of Holant $([0,1,1,0] \mid(=3))$ and Holant $\left(\frac{1}{4}[3,0,-1,0] \mid[2,0,2,0]\right)$ on the same instance graph $G$ are exactly the same by Valiant's Holant Theorem [16], as

$$
[0,1,1,0]\left(H^{-1}\right)^{\otimes 3}=\frac{1}{4}[3,0,-1,0] \quad \text { and } \quad H^{\otimes 3}(=3)=[2,0,2,0] .
$$

Notice that this is a many-to-many transformation among solutions of one problem Holant $\left([0,1,1,0] \mid\left(={ }_{3}\right)\right)$ (subsets of hyperedges that cover every vertex with no vertex covered three times) and another Holant $\left(\frac{1}{4}[3,0,-1,0] \mid[2,0,2,0]\right)$.

Next we will use the following gadgets, called matchgates, to "implement" the constraint functions $\frac{1}{4}[3,0,-1,0]$ and $[2,0,2,0]$ by perfect matchings. Consider the following two matchgates.

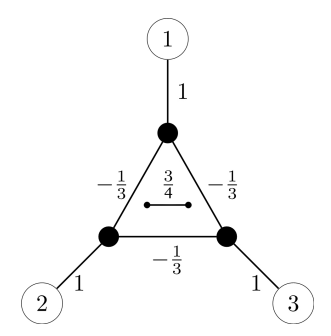

(a) Matchgate for $\frac{1}{4}[3,0,-1,0]$

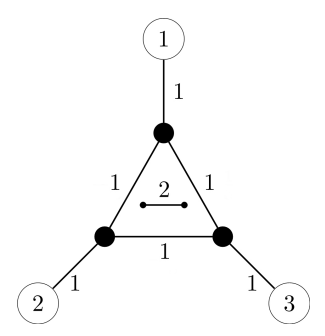

(b) Matchgate for $[2,0,2,0]$

Figure 2: Matchgates

For the matchgate in Figure 2 (a), we consider its perfect matchings, when any subset $\emptyset \subseteq$ $S \subseteq\{1,2,3\}$ of the external nodes labeled 1,2,3 are removed. For $S=\emptyset$ there is a unique perfect matching $M$ with weight $w(M)=3 / 4$. If $|S|=2$, we get $w(S)=-1 / 3$. If $|S|$ is odd, then $w(S)=0$. In this sense the matchgate has the symmetric signature $\frac{1}{4}[3,0,-1,0]$.

A similar calculation shows that the the matchgate in Figure 2 (b), has the symmetric signature $[2,0,2,0]$.

Now we take a planar input bipartite graph $G$ for Pl-Holant $([0,1,1,0] \mid(=3))$, replace each vertex of degree three on the LHS (which has the label $[0,1,1,0]$ ) by the matchgate in Figure 2 (a), replace each vertex of degree three on the RHS (which has the label $[1,0,0,1]$ ) by the matchgate in 
Figure 2 (b), and for any edge in $G$ add one edge with weight 1 between the corresponding external vertices from their respective matchgates. This creates a planar graph $G^{\prime}$, of size linearly bounded by that of $G$.

A moment reflection should convince the reader that the value $\# \operatorname{PM}\left(G^{\prime}\right)$ is exactly the same as the Holant value Holant $(G)$.

\section{References}

[1] Miriam Backens. A complete dichotomy for complex-valued Holant ${ }^{c}$. In Ioannis Chatzigiannakis, Christos Kaklamanis, Dániel Marx, and Donald Sannella, editors, 45th International Colloquium on Automata, Languages, and Programming, ICALP 2018, July 9-13, 2018, Prague, Czech Republic, volume 107 of LIPIcs, pages 12:1-12:14. Schloss Dagstuhl - LeibnizZentrum für Informatik, 2018.

[2] Miriam Backens and Leslie Ann Goldberg. Holant clones and the approximability of conservative Holant problems. ACM Trans. Algorithms, 16(2):23:1-23:55, 2020.

[3] Andrei A. Bulatov. The complexity of the counting constraint satisfaction problem. J. ACM, 60(5), October 2013.

[4] Jin-Yi Cai and Xi Chen. Complexity Dichotomies for Counting Problems: Volume 1, Boolean Domain. Cambridge University Press, 2017.

[5] Jin-Yi Cai and Xi Chen. Complexity of counting CSP with complex weights. J. ACM, 64(3):19:1-19:39, 2017.

[6] Jin-Yi Cai, Xi Chen, and Pinyan Lu. Nonnegative weighted \#CSP: An effective complexity dichotomy. SIAM J. Comput., 45(6):2177-2198, 2016.

[7] Jin-Yi Cai and Artem Govorov. Perfect matchings, rank of connection tensors and graph homomorphisms. In Timothy M. Chan, editor, Proceedings of the Thirtieth Annual ACMSIAM Symposium on Discrete Algorithms, SODA 2019, San Diego, California, USA, January 6-9, 2019, pages 476-495. SIAM, 2019.

[8] Jin-Yi Cai, Heng Guo, and Tyson Williams. A complete dichotomy rises from the capture of vanishing signatures. SIAM Journal on Computing, 45(5):1671-1728, 2016.

[9] Martin Dyer and David Richerby. An effective dichotomy for the counting constraint satisfaction problem. SIAM Journal on Computing, 42(3):1245-1274, 2013.

[10] M. Freedman, L. Lovász, and A. Schrijver. Reflection positivity, rank connectivity, and homomorphism of graphs. J. Amer. Math. Soc., 20(1):37-51, 2007.

[11] Teofilo F. Gonzalez. Clustering to minimize the maximum intercluster distance. Theoretical computer science, 38:293-306, 1985.

[12] Michael Kowalczyk and Jin-Yi Cai. Holant problems for 3-regular graphs with complex edge functions. Theory Comput. Syst., 59(1):133-158, 2016. 
[13] Jiabao Lin and Hanpin Wang. The complexity of boolean holant problems with nonnegative weights. SIAM Journal on Computing, 47(3):798-828, 2018.

[14] Shuai Shao and Jin-Yi Cai. A dichotomy for real boolean holant problems. To appear in FOCS 2020 arXiv preprint arXiv:2005.07906, 2020.

[15] Salil P. Vadhan. The complexity of counting in sparse, regular, and planar graphs. SIAM Journal on Computing, 31(2):398-427, 2001.

[16] Leslie G Valiant. Holographic algorithms. SIAM Journal on Computing, 37(5):1565-1594, 2008. 